\title{
A Novel LNP-based Chlamydia subunit vaccine formulation that induces Th1 responses without upregulating IL-17 provides equivalent protection in mice as formulations that induced IL-17 and Th1 cytokines
}

Melissa Ann Boddicker ( $\square$ melissa_wooters@merck.com )

Merck \& Co Inc https://orcid.org/0000-0002-5944-1738

Robin M. Kaufhold

Merck \& Co Inc

Kara S. Cox

Merck \& Co Inc

Bob J. Lucas

Merck \& Co Inc

Jinfu Xie

Merck \& Co Inc

Deborah D. Nahas

Merck \& Co Inc

Sinoeun Touch

Merck \& Co Inc

Amy S. Espeseth

Merck \& Co Inc

Kalpit Vora

Merck \& Co Inc

Julie M. Skinner

Merck \& Co Inc

Research article

Keywords: LNP, Chlamydia, IL-17, mouse model, Th1 cytokines

Posted Date: November 4th, 2019

DOl: https://doi.org/10.21203/rs.2.16752/v1 
License: (c) (i) This work is licensed under a Creative Commons Attribution 4.0 International License. Read Full License 


\section{Abstract}

Sexually transmitted Chlamydia infections can lead to the development of debilitating diseases such as chronic pelvic pain, ectopic pregnancy, pelvic inflammatory disease, and infertility. It has been proposed that immunity against Chlamydia infection and disease may be determined by a balance of IL-17 signaling. We sought to evaluate novel formulations for a candidate Chlamydia vaccine, consisting of Chlamydia major outer membrane protein (MOMP) alone or in combination with polymorphic membrane protein $\mathrm{D}(\mathrm{PmpD})$ and polymorphic membrane protein $\mathrm{G}(\mathrm{PmpG})$ as target immunogens. Native MOMP (nMOMP) isolated from C. muridarum elementary bodies (EBs) and recombinant PmpD and PmpG proteins were adjuvanted with Monophosphoryl lipid A (MPLA), in one of two formulations containing either lipid nanoparticles (LNPs) or the cationic lipid dimethyldioctadecylammonium bromide (DDA). Antibody titers to C. muridarum, nMOMP, and EBs were evaluated by ELISA, and T-cell responses by intracellular cytokine staining (ICS). Protection from challenge was determined by qPCR and gross pathology. All mice immunized with the new vaccine formulations showed significantly higher antibody titers to nMOMP $(P<0.001)$ and $C$. muridarum EBs $(P<0.001)$, when compared to the negative control group (adjuvant alone). Antibody titers in vaccine groups with Monophosphoryl lipid A (MPLA)+LNP were higher as compared to the MPLA+DDA group $(P<0.001)$ except for groups $6(\mathrm{Cm} n M O M P+P m p G+P m p D$ p73+PmpD p82+MPLA+DDA) vs 7 (Cm nMOMP+PmpG+PmpD p73+PmpD p82+MPLA+LNP) for both C. muridarum EBs and PmpG; the groups were not statistically significant. ICS analysis showed more robust CD4+ T-cell responses (IFN- - /LL-2/TNF- $a$ ) in the dimethyldioctadecylammonium bromide (DDA) and LNP groups compared to the adjuvant alone group. The combination of DDA and MPLA gave robust Th17 responses in comparison to MPLA and LNP group. Immunized groups also showed protection from challenge with $\mathrm{C}$. muridarum, as evidenced by a reduction in bacterial shedding from the vagina for all groups $(P<0.003)$ compared to shedding from the adjuvant control, Group 1. Both vaccine formulations generated robust immunological responses and both vaccine formulations were protective by reducing bacterial shedding after challenge. This data indicates equal protection can be achieved without the induction of Th17 responses.

\section{Background}

Chlamydia infections cause substantial morbidity and socio-economic burden world-wide, and although antibiotic treatment is effective, the high rate of asymptomatic infections ( $70 \%$ in women) leads to chronic infections that may ultimately cause upper genital tract pathology. An efficacious vaccine could prevent the disease sequalae that are common following Chlamydia infections including infertility and ectopic pregnancy (1-3). Each year approximately 1.4 million new cases of sexually transmitted $C$. trachomatis infections are diagnosed in the United States and approximately 113 million new cases are reported worldwide $(2,4)$. Although $C$. trachomatis infection is responsive to antibiotic treatment, more than $70 \%$ of infections in women are asymptomatic (5), making both diagnosis and control of infection difficult. Therefore, there is a substantial need for an efficacious vaccine to protect against Chlamydia infection. 
Chlamydia vaccine development has been decades long endeavor. In recent years encouraging progress has been made towards a native MOMP based vaccine with levels of protection approaching those of live $E B$ vaccination in mice (6-8). An antigen based subunit vaccine, whether using native purified proteins or recombinantly expressed proteins appears as a practical path forward. Although MOMP is a promising vaccine target, additional antigens may need to be included to create an efficacious vaccine.

Recent vaccine studies using MOMP and Pmp combinations have shown promise as vaccine candidates with early clearance of Chlamydia infections compared with unvaccinated control groups (9-12). The Pmp family of proteins are good T-cell antigens and potential targets for a T-cell focused vaccine $(9,11$, 13). Native MOMP has been shown to elicit a strong humoral immune response (14). A vaccine that includes antigens that provide both a T-cell and antibody response against Chlamydia may provide optimal protection against both initial and repeat infection than a vaccine profile that only elicits a Th1 or Th2 responses (11). IL -17 mediated immunity has been shown to play a role in the control of intracellular pathogens, including Chlamydia $(10,15)$ However, there is also evidence for a pathogenic role through enhanced recruitment of polymorphonuclear neutrophils and inflammatory monocytes in the UGT. The Th17-mediated inflammation has been associated with tissue destruction, intra-abdominal adhesions and increases in splenic weights in the mouse model (15-17). We tested the role of Th1 and/or Th17 Tcells in affording protection from Chlamydia infection and their ability to cause pathology in the upper genital tract utilizing two vaccine formulations.

We have evaluated numerous conserved Chlamydia antigen targets (data not shown) with minimal protection or reduction in bacterial burden observed following immunization and intravaginal challenge from most targets. Interestingly, we have observed protection with vaccine formulations including nMOMP similar to what has been published in the literature $(6-8,18)$. Since MOMP is a good humoral antigen, we also looked for antigens that would elicit cell-mediated immune responses. Immunoproteomic vaccine studies found that four polymorphic membrane proteins (PmpE, PmpF, PmpG and PmpH) are immunodominant, recognized by various $\mathrm{MHC}$ class II haplotypes and are protective in mouse models (9-11). PmpG/F have been shown to be the most immunodominant of the Pmps (11). Therefore, based on protection with nMOMP observed in our laboratory; and publications citing protective immunity for both MOMP and polymorphic membrane proteins, with PmpG/F being most immunodominate of the pmps, we focused on nMOMP, PmpG, PmpD passenger domain (PmpD-p73) and PmpD translocator domain (PmpD-p82) as candidate vaccine targets in the current study. Additionally, various adjuvants were included to drive the cell mediated immune response toward Th1, and/or Th17 and the role of Thelper population in conferring protection was evaluated. To determine the role of Th17 mediated immunity, vaccine formulations in some test groups were adjuvanted with the cationic lipid dimethyldioctadecylammonium bromide (DDA) which has been shown to induce potent Th1 and Th17 responses (19) whereas the lipid nanoparticles (LNP) formulations were used to bias toward Th1 responses. Immunogenicity was evaluated for both T-cell responses and antibody, and efficacy was determined by measuring the magnitude and duration of shedding of Chlamydia after intravaginal challenge. The combination of DDA and MPLA gave robust Th17 responses in comparison to LNP and MPLA. Both sets of formulated antigens (MPLA+LNP or MPLA+DDA) resulted in robust antibody titers to 
nMOMP $(P<0.001)$ and $C$. muridarum EBs $(P<0.001)$, when compared to the adjuvant-alone control group. Immunized groups also showed protection from challenge with $C$. muridarum, as evidenced by a reduction in bacterial shedding from the vagina for all groups $(P<0.003)$ compared to shedding from the adjuvant control, Group 1. Taken together, the data suggest that the vaccines formulated with either MPLA+LNP or MPLA+DDA elicited strong immunological responses and both were protective by reducing bacterial shedding after challenge. Furthermore, equal protection is achieved with or without a Th17 response.

\section{Methods}

\section{Ethics statement}

All animal experiments were approved by the Merck \& Co., Inc., Kenilworth, NJ, USA, Institutional Animal Care and Use Committee (IACUC). All procedures were performed in accordance with our institution's IACUC guidelines in strict accordance with the recommendations in the Guide for Care and Use of Laboratory Animals of the National Institutes of Health. Mice were housed in large mouse containers $(\mathrm{n}=$ 10 mice/box and $n=5$ mice/box for ICS) with micro isolator lids, and the rooms were maintained with controlled humidity and temperature, and 12-hour light-dark cycles. All containers had nestlets and animals were provided standard chow (Purina 5001 rodent diet) and water ad libitum. The physical condition of the animals was monitored daily (7 days a week), and any health changes were noted. At the end of the study, animals were euthanized using $\mathrm{CO} 2$ inhalation $(10 \%-30 \% \mathrm{CO} 2$ in the air mixture inhaled per minute). No animals were found to be moribund or to have any adverse advents throughout the study.

\section{Cell culture and propagation of Chlamydia muridarum}

C. muridarum was obtained from Bernard Arulanandam (UTSA San Antonio, Texas). HeLa 229 cells were used for propagation of $C$. muridarum. HeLa 229 cells were grown in Eagle's Minimal Essential Medium (EMEM; ATCC, Manassas, VA) supplemented with 10\% heat-inactivated fetal bovine serum (FBS; Hyclone, Logan, UT), $50 \mathrm{gg} / \mathrm{ml}$ vancomycin (Sigma, St. Louis, MO), and $10 \mathrm{gg} / \mathrm{ml}$ gentamicin (Gibco; Carlsbad, CA). Host cells were seeded into tissue culture flasks at a cell density of $5 \times 10^{5}$ cells $/ \mathrm{ml}$ and incubated overnight at $37^{\circ} \mathrm{C}$ in $5 \% \mathrm{CO}_{2}$ to achieve a confluent monolayer. Cell monolayers were infected with a Chlamydia stock diluted in sucrose-phosphate-glutamate (SPG) buffer. The $C$. muridarum strain was cultured for 48 hours. The Chlamydiae were harvested from the infected cells and purified by centrifugation through $30 \%$ Renograffin (Bracco Diagnostics, Princeton, $\mathrm{NJ}$ ) and stored frozen at $-80^{\circ} \mathrm{C}$.

\section{Chlamydia recombinant proteins}

The recombinant Chlamydia proteins were cloned, expressed and purified as follows: PmpG (amino acids 25-500), PmpD passenger domain (p73) (amino acids 53-757) and PmpD translocator domain (p82) (amino acids 758-1520) as described by Swanson (20); C. muridarum gene sequences were synthesized by Geneart (Thermo Fisher, Germany) and cloned into pET30a and pET28a vectors (Novagen, Cambridge, MA), respectively, using standard molecular biology techniques. Plasmids containing the 
genes were transformed into the Escherichia coli strain BL21(DE3) (New England Biolabs, Ipswich, MA) with protein expression induced using the lac promotor for T7 RNA expression using isopropyl-B-Dthiogalactopyranoside (IPTG).

\section{Purification of His-tagged PmpG, PmpD-p73 and PmpD-p82}

Cell pellets of E. coli expressing PmpG, PmpD-p73 or PmpD-p82 were resuspended in lysis buffer (50 mM TRIS, pH 8.0, $300 \mathrm{mM} \mathrm{NaCl}$, and 1X protease inhibitors (Roche, Basel, Switzerland, EDTA free), and lysed with three passes through a microfluidizer (Microfluidics M-110P) at 15,000psi. The lysate was clarified by centrifugation $(\sim 19,000 \mathrm{xg})$ and the pellet (containing inclusion bodies) was solubilized in a urea buffer ( $8 \mathrm{M}$ urea, $10 \mathrm{mM}$ MES pH $6.5,150 \mathrm{mM} \mathrm{NaCl}$, and $20 \mathrm{mM}$ Imidazole) and clarified by ultracentrifugation ( 160,000xg). The solubilized fraction was purified while in urea by immobilized metal affinity chromatography (IMAC) with Ni NTA agarose and step eluted with $200 \mathrm{mM}$ imidazole. The IMAC product was refolded using extensive dialysis against $10 \mathrm{mM} \mathrm{MES} \mathrm{pH} \mathrm{7.3,150mM} \mathrm{NaCl} \mathrm{containing} 0.1 \%$ Zwittergent, 3-14 (PmpD-p73 and PmpD-p82) or phosphate buffered saline (PBS) containing $0.1 \%$ Zwittergent, 3-14 (PmpG) using a Pierce Slide-A-Lyzer (Pierce, Rockford, Illinois). cassette with a $10 \mathrm{kDa}$ MWCO while step reducing the urea concentration to zero. The PmpD-p73 and PmpD-p82 in the MES buffered saline containing $0.1 \%$ Zwittergent, $3-14$ or the PmpG in the PBS containing $0.1 \%$ Zwittergent, 3-14 were used in both in vitro and in vivo studies. The final products were sterile filtered and the concentration was determined by amino acid analysis. Purity of the final product was evaluated by SDSPAGE and by in-solution enzymatic digestion with1D/LC-MS-MS analysis.

\section{Amino Acid Analysis}

Vapor phase hydrolysis was performed with constant boiling $6 \mathrm{~N} \mathrm{HCl}$ at $150{ }^{\circ} \mathrm{C}$ for 1 hour using an ELDEX (Napa, CA) H/D workstation. Protein hydrolysates and amino acid standards were derivatized with 6aminoquinolyl-N-hydroxysuccinimidyl carbamate (AQC) using the Waters AccQ Tag Ultra derivatization kit according to the manufacturer's protocol. AQC reacts with primary and secondary amines to form stable fluorescent derivatives that can be separated by RP-HPLC. The fluorescent AQC derivatives were separated on an AccQ Tag Ultra C18, $1.7 \mu \mathrm{m}, 2.1 \times 100 \mathrm{~mm}$ column using a modified gradient program.

\section{Native MOMP purification from infected cells (HeLa)}

Native MOMP (nMOMP) was purified by extraction using sonication of intact Chlamydia-infected HeLa cells based on a method by de La Maza (18) with modifications to infected cell starting material, less frequent sonication, and altered chromatography. The initial steps used phosphate buffer with CHAPS and 100mM DTT and subsequently a phosphate buffer containing Zwittergent, 3-14 with 100mM DTT. The MOMP was further purified post-extraction with hydroxyapatite chromatography. The hydroxyapatite product was concentrated using tangential flow filtration (TFF), and then purified with anion exchange chromatography. The nMOMP was dialyzed into $10 \mathrm{mM} \mathrm{MES} \mathrm{pH} \mathrm{7.3,150mM} \mathrm{NaCl}$, and $0.1 \%$ Zwittergent, 3-14 for use in animal dosing and in vitro studies. 
Female C57BL/ 6 mice were purchased from Taconic Farms (Hudson, NY). At the time of study start, mice were 6-7 weeks of age, with food and water provided ad libitum. Animals were randomly assigned to cages upon receipt from the vendor. All animal experiments in these studies were approved by the Institutional Animal Care and Use Committee (IACUC), Merck \& Co., Inc., West Point, PA, USA. All procedures were performed in accordance with our institution's IACUC guidelines in strict accordance with the recommendations in the Guide for Care and Use of Laboratory Animals of the National Institutes of Health. Animals were treated and assessed in numerical order groups 1-8.

\section{Murine model and specimen collection}

Study design is shown in Figure 1. Fifteen animals per group (10 for challenge and 5 for ICS) were immunized by subcutaneous (s.c.) route with the following combinations of adjuvants and antigens as seen in Table 1: Group 1) MPLA+ DDA+ LNP (adjuvant control), Group 2) Cm nMOMP+MPLA+DDA, Group 3) $\mathrm{Cm}$ nMOMP+MPLA+LNP, Group 4) $\mathrm{Cm}$ nMOMP+PmpG+MPLA+DDA, Group 5) $\mathrm{Cm}$ nMOMP+PmpG+MPLA+LNP, Group 6) $\mathrm{Cm}$ nMOMP+PmpG+PmpD p73+PmpD p82+MPLA+DDA, Group 7) Cm nMOMP+PmpG+PmpD p73+PmpD p82+MPLA+LNP, Group 8) Live Cm EB (positive control) were immunized with $1 \times 10^{6} \mathrm{~EB}$ in SPG per mouse by intraperitoneal (i.p.) route.

Table 1. Vaccine Groups

\begin{tabular}{|l|l|l|l|l|l|}
\hline Group & Vaccine & $\begin{array}{c}\text { Antigen Dose } \\
(\mathrm{ug})\end{array}$ & MPLA Dose & DDA Dose & LNP Dose \\
\hline 1 & MPLA+DDA+LNP & - & $5 \mathrm{ug}$ & $250 \mathrm{ug}$ & $100 \mathrm{ug}$ \\
\hline 2 & Cm nMOMP+MPLA+DDA & 10 & $5 \mathrm{ug}$ & $250 \mathrm{ug}$ & - \\
\hline 3 & Cm nMOMP+MPLA+LNP & 10 & $5 \mathrm{ug}$ & - & $100 \mathrm{ug}$ \\
\hline 4 & Cm nMOMP+PmpG+MPLA+DDA & $10 / 10$ & $5 \mathrm{ug}$ & $250 \mathrm{ug}$ & - \\
\hline 5 & Cm nMOMP+PmpG+MPLA+LNP & $10 / 10$ & $5 \mathrm{ug}$ & - & $100 \mathrm{ug}$ \\
\hline 6 & $\begin{array}{c}\text { Cm nOMP+PmpG+PmpD } \\
\text { p73+PmpD p82+MPLA+DDA }\end{array}$ & $10 / 5 / 5 / 5$ & $5 \mathrm{ug}$ & $250 \mathrm{ug}$ & - \\
\hline 7 & $\begin{array}{c}\text { Cm nOMP+PmpG+PmpD } \\
\text { p73+PmpD p82+MPLA+LNP }\end{array}$ & $10 / 5 / 5 / 5$ & $5 \mathrm{ug}$ & - & $100 \mathrm{ug}$ \\
\hline 8 & Cm EBs & $1 \mathrm{x} 10^{\wedge} 6 \mathrm{IFU}$ & - & - & - \\
\hline
\end{tabular}

Tail-vein bleeds were performed on days -1 and day 42 . Immunizations were performed on days 0,20 , 30. Spleens were harvested on day 40 from 5 mice per group. Mice were rested for 1 month prior to intravaginal challenge. Progesterone [medroxyprogesterone acetate (Depo-Provera; Pfizer; New York, NY)] was administered subcutaneously ( $2.5 \mathrm{mg} / \mathrm{dose})$ on days 45 and 52 of the study prior to challenge. Mice were anesthetized in their home cage prior to challenge only with a mixture of $85 \mathrm{mg} / \mathrm{kg}$ ketamine (Ketaset; Fort Dodge Animal Health, Fort Dodge, IA) and $5 \mathrm{mg} / \mathrm{kg}$ xylazine (AnaSed $\circledast$ Injection; Henry Schein Animal Health, Dublin, $\mathrm{OH}$ ) by i.proute. Mice were challenged intravaginally by direct instillation of $5,000 \mathrm{IFU} C$. muridarum in $10 \mu \mathrm{I} \mathrm{SPG}$ on Day 55. The course of infection was monitored by enumerating Chlamydiae recovered from cervicovaginal swabs collected on days $62,69,76$. The vaginal vault and 
ectocervix were swabbed using a microfiber swab (Fisher; Pittsburgh, PA). Swabs were placed into a 1.5 $\mathrm{ml}$ tube containing two sterile glass beads ( $5 \mathrm{~mm}$ diameter) and $300 \mu \mathrm{l}$ of Chlamydia Isolation Medium (Trinity Biotech; Berkeley Heights, $\mathrm{NJ}$ ) on ice. Bacteria were eluted from the swabs and separated from cells by vortexing for 60 seconds. Eluted bacteria were split into two aliquots and stored at $-70^{\circ} \mathrm{C}$ until DNA extraction.

At spleen harvest or at the end of the study, animals were euthanized using $\mathrm{CO} 2$ inhalation $(10 \%-30 \%$ $\mathrm{CO} 2$ in the air mixture inhaled per minute). No animals were found to be moribund throughout the study. Gross pathology was assessed on day 118 .

\section{Primer, probe and real-time quantitative PCR}

The qPCR assays were performed as described in Wooters et al. (21), with the following modifications. Briefly, DNA from genital swab samples $(\sim 100 \mu \mathrm{l})$ was extracted using the MagNA Pure 96 DNA and Viral NA small volume kit (Roche, Basel, Switzerland) on the MagNA pure machine (Roche, Basel, Switzerland) according to the manufacturer's instructions. The oligonucleotide primer set was designed for detection of all species of Chlamydiae. The sense primers, 16S DIR 5'-ATG CAT ATG ACC GCG GCA A-3', and 16S DIR 5'-ATG TAT ATG ACC GCG GCA GA- 3'and anti-sense primer, 16S Rev 5'-TAA GGG CCA TGC TGA CTT GA-3', were designed to amplify a 508-bp fragment of the Chlamydial 16S ribosomal subunit gene, conserved across Chlamydia strains and serovars. Primers were obtained from Sigma-Aldrich (St. Louis, MO), and the probe, 16S Fam-5'-TTA ACC AGG AGG AAG GCG AG-3' Tamra, was also synthesized by Sigma-Aldrich (St. Louis, MO). The 50- $\mu \mathrm{L}$ reaction mixtures consisted of $1 \mathrm{X}$ QuantiTect Multiplex PCR master mix without ROX (Qiagen, Hilden, Germany), $100 \mathrm{nmol} / \mathrm{L} 16 \mathrm{~S}$ probe, $200 \mathrm{nmol} / \mathrm{L}$ primer 16S DIR, $400 \mathrm{nmol} / \mathrm{L}$ primer $16 \mathrm{~S} \mathrm{Rev}, 30 \mathrm{nmol} / \mathrm{L}$ ROX reference dye, and $5 \mu \mathrm{L}$ of sample DNA. Nontemplate controls consisting of the reaction master mix, primers, and probe, but no DNA, were included in each assay run. Reaction conditions were set as follows: 1 cycle at $95^{\circ} \mathrm{C}$ for $15 \mathrm{~min}$, followed by 40 cycles at $94^{\circ} \mathrm{C}$ for $1 \mathrm{~min}$ and at $60^{\circ} \mathrm{C}$ for $1 \mathrm{~min}$. Thermal cycling, fluorescent data collection, and data analysis were performed using the Stratagene Mx3005P system (Stratagene, San Diego, CA) according to the manufacturer's instructions. Data were plotted as Area Under the Curve (AUC) for individual mice and further analyzed by Wilcoxon Rank Sum Test (compared to MPLA / DDA / LNP).

\section{Detection of serum antibody and isotype levels by ELISA}

Serum was analyzed by an enzyme-linked immunosorbent assay (ELISA). Nunc ${ }^{\mathrm{TM}}$ C96 Maxisorp Immunoplates (Thermo Scientific, Waltham, MA) were coated with $50 \mu \mathrm{L}$ of $1 \mu \mathrm{g} / \mathrm{ml}$ C. muridarum EBs in PBS at $4^{\circ} \mathrm{C}$ overnight. The plates were washed three times with PBS containing $0.05 \%$ Tween -20 (Fisher Scientific, Hampton, NH)(PBS-T). The wells were blocked with 5\% Fetal Bovine Serum Albumin (BSA) (Thermo Scientific, Waltham, MA) in PBS-T at $200 \mu \mathrm{L} /$ well for 1 hour at room temperature and washed three times with PBS-T. Serum was diluted in 5\% FBS in PBS at a 1:500 dilution. Serially diluted sera were added to the plate, incubated for 2 hours at room temperature and were washed three times with PBS-T. HRP-conjugated secondary antibodies (Goat anti-mouse IgG, Fcy fragment specific 1:6,000; Goat antimouse IgG, Fcy Subclass 1 specific 1:6,000; or Goat anti-mouse IgG, Fcy Subclass 2c specific 1:2,000; 
Jackson ImmunoResearch Laboratories, Grove, PA) were diluted in 5\% FBS in PBS. The diluted secondary antibodies were added at $100 \mu \mathrm{L} /$ well, incubated for 1 hour at room temperature and were washed three times with PBS-T followed by three times with PBS. Room temperature BD Opt EIA ${ }^{\mathrm{TM}}$ TMB Substrate Reagent Set (BD Biosciences, Franklin Lakes, $\mathrm{NJ}$ ) was mixed and filtered through a 0.22um cellulose acetate (CA) filter unit (Corning, Corning NY), and $100 \mu \mathrm{L}$ was added to each well and incubated for 10 min at room temperature. The reaction was stopped with $100 \mathrm{\mu L} /$ well of $2 \mathrm{M} \mathrm{H}_{2} \mathrm{SO}_{4}$ (Fisher Scientific, Hampton, NH). The optical density (OD) was read at $450 \mathrm{~nm}$ on a SpectraMax® M5 (Molecular Devices, San Jose, CA). The cutoff OD for each post-immunization serum was calculated as two times of the $\mathrm{OD}_{450}$ of the corresponding pre-immunization serum. ELISA titers were determined by linearly interpolating between the sequential log dilutions that bracket the cutoff $O D$, where the dependent variable is the $\mathrm{OD}$ response and the independent variable is the log dilution. The resulting dilution is then back transformed to obtain the reported titer. The reported titer is the estimated dilution of serum that results in a response equivalent to the endpoint OD. Transformed was data analyzed by One-way ANOVA with Dunnett post test

\section{T-cell intracellular cytokine staining}

Ten days post final immunization, spleens from vaccinated animals were collected and processed to isolate splenocytes. One million splenocytes/well were incubated with $2 \mu \mathrm{g} / \mathrm{ml}$ of specific peptide pools for MOMP, PmpG or PmpD (15mer overlapping by 11, custom order JPT Peptide Solns, Germany) in the presence of Brefeldin A and anti-mouse CD28 and CD48b antibodies (BD Biosciences, Franklin Lakes, $\mathrm{NJ}$ ). As a negative control, a sample for each was set up with a matched volume of DMSO and costimulatory antibodies, 5 hours post incubation at $37^{\circ} \mathrm{C}$, splenocytes were incubated with mouse FC block (BD) surface stained with viability dye (Thermo Fisher, Waltham, MA) anti-mouse-CD3 (BD), CD4 (BD), CD8 (BD) followed by permeabilization and intracellular staining for anti-mouse IFNY (BD), TNFa (BD), IL-2 (BD), and IL-17A (Biolegend), San Diego, CA). Fixed samples were then run on FACS LSRII flow cytometer (BD Biosciences, Franklin Lakes, NJ) and data were analyzed using FloJo software (Treestar Inc., Ashland, OR). The data analysis program Simplified Presentation of Incredibly Complex Evaluations (version 4.1.6; provided by M. Roederer, Vaccine Research Center, NIAID, NIH) was used to generate the graphical results in Figure 2. All peptide stimulated responses were reported after subtraction of the unstimulated controls. Responses were compared to the adjuvant only group (MPLA+DDA+LNP) in a two tailed paired $t$ test.

\section{Results}

\section{Recombinant and native protein purification and analytical evaluation}

PmpD (separated into the passenger domain p73 and tranlocator domains p82) and PmpG were cloned and expressed using $\mathrm{pET}$ vector systems. The recombinant proteins were expressed in the inclusion body fraction of IPTG-induced BL21DE3 cells. The inclusion body fractions were denatured and recombinant proteins were purified and refolded (made soluble) using dialysis refolding techniques that provided high 
purity proteins for in vitro and in vivo use. All antigens tested had purities of $>99 \%$ by 1D/LC-MS-MS analysis and were maintained in buffers (PBS with $0.1 \%$ Zwittergent,3-14 or $\mathrm{MES} / \mathrm{NaCl}$ with $0.1 \%$ Zwittergent,3-14) that were suitable for both in vitro and in vivo studies. Native MOMP (nMOMP) was purified from large scale preps of Chlamydia muridarum infected HeLa cells. The nMOMP materials used in these studies were analyzed by amino acid analysis to determine concentration and then by 1D/LC-MSMS analysis (purity $>95 \%$ ), size exclusion chromatography with multi-angle light scattering detection to determine structure (predominantly trimer with some monomer), circular dichroism to determine form ( 40\% Beta-sheet structure), and SDS-PAGE analysis to confirm purity and structural characteristics (data not shown).

Immunization with Chlamydia proteins adjuvanted with LNP or DDA induced robust and distinct polyfunctional CD4+ T-cell response profiles, and IL-17 did not enhance protection from challenge

Due to the well-established connection between CD4+ T-cells and control of Chlamydia, we included potent T-cell adjuvants in our vaccine formulations (22-25). To explore the role of IL-17 in protection from Chlamydia challenge, we included formulations containing the cationic lipid, DDA, which was shown to be a potent promoter of IL-17 in previous vaccine studies (10)). Multiparameter flow cytometry was performed to evaluate antigen specific IFN- $y$, TNF-, IL-2 and IL-17 producing T-cells in splenocytes ten days after the final immunization (d40). Five mice per group were analyzed for ICS.

Robust MOMP specific CD4+ T-cells were observed in groups vaccinated with adjuvanted nMOMP (Figure 2). Furthermore, MOMP responses of similar magnitude were detected from mice that were vaccinated with additional proteins (PmpG, PmpD) (Figure 2), demonstrating a lack of immune interference.

Strong CD4+ T-cell responses specific for PmpD or PmpG were also detected in mice vaccinated with the respective adjuvanted proteins (Figure 2). Surprisingly, despite the increased protection afforded by the intraperitoneal C. muridarum elementary body immunization (Figure 4), mice in these groups demonstrated low levels of CD4+ T-cell responses in the spleen when stimulated with MOMP, PmpD and PmpG peptides. Compared to the adjuvant only control group, significant T-cell responses (IFN- $y$ and TNF-) in EB exposed mice were only detected towards MOMP and PmpG (Figure 2). Additionally, these responses were much lower in magnitude as compared with the subcutaneous protein vaccinated groups.

As expected, potent IL-17 responses were detected from mice in groups that were vaccinated with DDA containing formulations (Figure 2D), and such responses were not induced by the LNP formulations or $C$. muridarum EB exposure. Additionally, IL-2 cytokine production trended higher in the groups which received DDA (Figure $2 \mathrm{C}$ ).

As polyfunctional T-cell responses have been shown to correlate with protection in many infectious disease models, we evaluated the percent of total responses in each group which produced all four cytokines (IFN-y/IL-2/TNF-/IL-17), any combination of the three, two or any one cytokine (Figure 2). Primarily due to significantly increased IL -17 and IL -2 production in the DDA adjuvanted vaccine groups 
(Figure 2C and 2D), a higher percentage of polyfunctional T-cells were detected in the DDA adjuvanted vaccine groups as compared to the LNP or EB exposed groups (Figure 2E).

\section{Vaccine immunizations result in high ELISA titers to $n M O M P$, C. muridarum EBs, PmpG and PmpD}

Animals were immunized with combinations of recombinant and native extracted proteins as per the scheme depicted in Table 1. An adjuvant control group with MPLA, DDA and LNP was used as a comparator against all test vaccine groups (10 animals per group). Each of the following combinations of antigens were formulated with MPLA+DDA or MPLA+LNP: nMOMP; nMOMP + PmpG; and nMOMP + PmpG + PmpD-p73 + PmpD-p82 (Table 1). A live Cm EB group was administered intraperitoneally as a positive control for protection against Chlamydia challenge $(26,27)$. Sera collected prior to primary immunization and on day 42 were tested in ELISAs for antibodies to nMOMP, C. muridarum EBs, PmpG, PmpD-p73 and PmpD-p82. Anti-nMOMP IgG titers were high for all vaccine groups with the MPLA/LNP adjuvanted groups $(3,5,7)$ inducing significantly higher $(P<0.001)$ IgG titers than MPLA/DDA adjuvanted groups $(2,4,6)$ (Figure $3 A$ ). The IgG1/lgG2c ratios for MPLA/LNP groups compared to MPLA/DDA groups were not statistically different, although the ratios indicate an overall balanced $\lg \mathrm{G} 1 / \mathrm{lgG} 2 \mathrm{c}$ responses for most vaccinated groups with the exception of group 2 (nMOMP + MPLA+ DDA), group 6 (containing all antigens: nMOMP, PmpG, PmpD-p73, PmpD-p82+MPLA+DDA) and group 8 (live EB), which were lower, favoring IgG2c. However, comparisons of group 1, 2, 6 or 8 were not statistically different from each other (data not shown). Groups with ratio's $<1$ indicate more IgG2C thus suggesting a bias towards Th1/IL -17 cytokine responses (Figure 3B). All vaccine groups had high IgG titers to $C$. muridarum EBs, with MPLA/LNP adjuvanted groups (groups 3 and 5) having statistically higher ELISA titers than MPLA/DDA adjuvanted groups (groups 2 and 4) (Figure 3C). Those groups containing PmpD-p73 and PmpD-p82 had high PmpD IgG titers, with the MPLA+LNP adjuvanted group (group 7) having approximately 1 to 3 logs higher titers than the MPLA+DDA adjuvanted group (group 6) (Figure 3D, 3E). Some cross reactivity to both PmpD-p73 and PmpD-p82 was observed for groups vaccinated with PmpG. Groups containing PmpG had high IgG titers to PmpG with the exception of group 6 (nMOMP/PmpG/PmpD p73/PmpD p82/ MPLA/DDA) which had a wider of titers against the Pmp antigens tested. Interestingly, PmpG antigens formulated with LNP, group 5 and 7, had higher titers than those formulated with DDA, groups 4 and 6 , although only group 4 and 5 were significantly different $(P<0.0001)$ (Figure $3 F)$.

Immunization with native and recombinantly expressed proteins resulted in early clearance of $C$. muridarum following genital tract infection

Following immunization, animals were challenged with $C$. muridarum intravaginally (10 animals per group). Bacterial burden was assessed by swabbing the vaginal vault and ectocervix. Samples were then evaluated via qPCR as described previously (21). Data were plotted as Area Under the Curve (AUC) for individual mice and further analyzed by Wilcoxon Rank Sum Test (compared to MPLA / DDA / LNP). All vaccine test groups with either adjuvant MPLA + DDA or MPLA + LNP had reduced bacterial burden and early clearance over time when compare to the adjuvant MPLA / DDA / LNP control group (Figure 4). The reduction in bacterial shedding from the vagina for all groups was statistically significant $(P<0.001)$, with 
Group 5 MOMP + PmpG + MPLA/LNP having a slightly lower statistical significance $(P=0.003)$. The EB positive control group was also protected from Chlamydia infection (Figure 4). Although the test vaccines appeared to decrease Chlamydia burden over the course of the infection, they failed to achieve the degree of protection provided by intraperitoneal administration of EBs. No statistical differences were observed between any of the vaccine groups (2-7). The EB positive control group was statistically lower than each of the vaccine test groups (2-7), p-values not shown.

\section{Discussion}

The development of an efficacious vaccine against Chlamydia is still an important unmet medical need. Since the first vaccine trials in humans more than 50 years ago, there have been valiant efforts to produce a vaccine against Chlamydia, to prevent both trachoma and genital tract infections. The early human vaccine trials produced valuable information regarding the lack of efficacy of formalin inactivated whole Chlamydia vaccines and the possible immunopathology that results from their use. Additionally, over the years, animal models have elucidated the protective immune responses necessary to clear both primary and secondary infections. There is hope that these animal models will inform us about the human disease and the findings will translate to protection in humans. The murine primary protective immune response has been found to be MHC-II restricted CD4+ T-cells with MHC-I restricted CD8+ T-cells playing a less critical role in protection (22-25). Antibodies will also be important in vaccine development as the protective role for antibodies has been demonstrated in relation to reinfection following a primary $C$. muridarum infection in mice (28-30). Cytokines of a Th1 profile such as IFN-y and interleukin 12 (IL-12) are essential against $C$. muridarum infection in mice $(31,32)$. In addition, IL-17 mediated immunity has been shown to play a role in the control of intracellular pathogens, including Chlamydia $(10,15)$. However, there is also some evidence for a pathogenic role for IL -17 through enhanced recruitment of polymorphonuclear neutrophils and inflammatory monocytes in the UGT. The Th17-mediated inflammation has been associated tissue destruction and with intra-abdominal adhesions and increases in splenic weights in the mouse model (15-17).

In this study we compared subunit vaccines of native extracted MOMP either alone or in combination with other recombinantly expressed outer membrane MHC-II restricted proteins (PmpG, PmpD-p73 and PmpDp82) to evaluate whether the addition of Pmp proteins would confer a greater reduction in bacterial shedding after challenge compared to nMOMP alone. In addition, we included various adjuvants to drive the cell mediated immune response toward Th1 and Th1/Th17 and to address the role of IL-17 in protection. The vaccines were adjuvanted with MPLA/DDA or MPLA/LNP and tested in C57BI/ 6 mice. MPLA elicits a strong Th1 and balanced IgG1/lgG2 response when formulated with protein vaccines. LNPs when co-mixed with some sub-unit antigens result in strong B-cell responses, CD4+ and CD8+ T-cell responses (33). The B-cell responses are typically Th2-like (based on IgG2a:IgG1 ratio). The antigen specific CD4+ and CD8+ T-cell responses boosted by LNP are typically polyfunctional: IFN- $\gamma+T N F-+I L-2+$ (33). DDA has been shown to increase Th1 responses yielding a mixed Th1:Th2 response and an increased IL-17+ response (34). 
High systemic antibody titers were seen overall in this study. The LNP+MPLA adjuvanted groups had higher antibody titers when compared to MPLA+DDA adjuvanted group. Interestingly higher titers of antibodies in the LNP+MPLA groups did not translate to better clearance of Chlamydia following the intravaginal challenge. There can be several potential explanations for this observation, including higher amplitude of serum antibody titer may not reflect the antibody titers in the mucosal compartment thus not translating into improved protection from vaginal challenge. Furthermore, the functionality of the antibodies raised could be different for the two groups of adjuvanted formulations. We plan to evaluate functional mucosal responses in future studies.

In these studies, we observed a reduction in overall bacterial shedding in vaccine test groups that were statistically better than the adjuvant control group. We observed that an increased Th17+ response did not add to the protective immune responses nor did the addition of any of the Pmp family of antigens to a native MOMP vaccine elicit an increase in protection following intravaginal challenge. Our data also indicated that eliciting Th1 responses in the absence of Th17 responses were equally protective as MPLA+LNP formulations were comparable to MPLA+DDA formulations in terms of reducing the Chlamydia load after challenge. These data are consistent with publications that have shown that the protective immune response to Chlamydia infection appears to be correlated with a dominant Th1 and low Th17 T-cell response (26). Mice in the DDA formulated groups did not have significantly lower bacterial shedding after challenge as compared to the mice in the LNP adjuvanted groups (Group 2 vs Group 3 p-value = 0.68, Group 4 vs Group5 p-value = 0.38, Groups 6 vs Group 7 p-value $=0.31$, Figure 4). This suggests that the ability of the DDA-formulated vaccines to elicit an IL -17 response did not confer greater protection from shedding than IFN- $\mathrm{Y}, \mathrm{IL}-2$ and TNF- type Th1 response in this challenge model.

No significant differences were observed in reduction of pathology for any of the groups including the live EB immunized group in this study (data not shown). Due to similar pathology observed across all adjuvanted groups and EB vaccinated group, the role of $\mathrm{IL}-17$ in inducing pathology could not be determined. Consistent to our observation, it has been reported that EBs immunized intranasally were more resistant to genital tract pathology than EBs immunized intraperitoneally in mice upon subsequent intravaginal Chlamydia challenge. This observation could be postulated to better mucosal immunity upon intranasal immunization compared to intraperitoneal vaccinations.(26). A good mucosal immune response may prevent Chlamydia ascension to the upper genital tract. Future studies could include for academic purposes, intranasal immunization with live EBs to test whether this immunization route would be more resistant to genital tract pathology consistent with the literature.

Our nMOMP immunized groups conferred protection from challenge similar to previous publications (6$8,18)$. For those groups combining MOMP and either PmpD or PmpG or a combination of both PmpD and PmpG (PmpDG), we did not see an increased reduction of bacterial shedding compared to nMOMP alone after intravaginal challenge. This data was in contrast to previous reports that showed the combination of Pmp E, F, G, H + MOMP reduced shedding over MOMP alone, PmpG alone, or the combination of Pmps (11). However, it should be noted that there are marked differences between the studies performed herein and those reported previously. Differences include the combination of Pmps, 
protein purification methods, study designs (antigen dose, adjuvants), assessment following challenge (sample collection, Chlamydia shedding assay), all of which may theoretically result in the lack of increased protection for the combination vaccine image. The Pmps in our study were selected based on recombinant protein expression and availability; a future study would need to include additional recombinantly expressed Pmps such as E, F, and $\mathrm{H}$. In addition to including more Pmps in a future study, a stricter alignment using the same protein purification methods, the use of same adjuvants, and assessment following challenge is warranted for a closer comparison between the data sets. Further, a direct comparison of the reagents and antigens generated from the previous studies with our reagents and vaccine combinations in a side by side comparison would be beneficial to elucidate why the results were not similar.

\section{Conclusions}

In conclusion, we found that Chlamydia vaccines based on nMOMP provided modest protection against intravaginal challenge with Chlamydia muridarum. The efficacy of these vaccines could not be improved either by the incorporation of additional Pmp antigens, or by formulating the nMOMP antigen with adjuvants that either increased circulating anti-nMOMP IgG, or by eliciting a Th17+ response to nMOMP. An efficacious vaccine will require finding the optimal combination of antigens with several targets included and an adjuvant/immunization route combination that results in multifunctional CD4+ T cells and humoral responses elicited systemically and in the mucosal compartments.

\section{Abbreviations}

IFU, inclusion forming unit; EB, elementary body; MOMP, Major Outer Membrane Protein; PmpG, polymorphic membrane protein G; PmpD polymorphic membrane protein D; intracellular cytokine staining (ICS), IL-17, Interlukin-17, MPLA Monophosphoryl lipid A, DDA dimethyldioctadecylammonium bromide, upper genital tract (UGT)

\section{Declarations}

Availability of data and materials

The datasets used and/or analyzed during the current study are available from the corresponding author on reasonable request.

\section{Competing interests}

All authors are employee of Merck \& Co., Inc., West Point, PA, USA

\section{Ethics Approval and Consent to Participate}

All animal experiments were approved by the Merck \& Co., Inc., Kenilworth, NJ, USA, Institutional Animal Care and Use Committee (IACUC). All procedures were performed in accordance with our institution's 
IACUC guidelines in strict accordance with the recommendations in the Guide for Care and Use of Laboratory Animals of the National Institutes of Health. Mice were housed in large mouse containers ( $\mathrm{n}=$ 10 mice/box) with micro isolator lids, and the rooms were maintained with controlled humidity and temperature, and 12-hour light-dark cycles. All containers had nestlets and animals were provided standard chow (Purina 5001 rodent diet) and water ad libitum. The physical condition of the animals was monitored daily (7 days a week), and any health changes were noted.

At the end of the study, animals were euthanized using CO2 inhalation (10\% -

$30 \% \mathrm{CO} 2$ in the air mixture inhaled per minute). No animals were found to be

moribund throughout the study.

\section{Authors' Contribution}

$\mathrm{MB}$ is the primary author, and performed recombinant protein cloning and expression, and performed additional data analysis for ELISAs and qPCR evaluations. RK conducted the animal studies and performed ELISAs and data analysis of the ELISAs. KC ran the FAQ assays and performed the data analyses and authoring of the related sections for FAQ analysis. BL purified both recombinant proteins and native Momp proteins. JX, purified Chlamydia and ran ELISAs and performed data analysis for ELISAs. DN performed amino acid sequence analysis; ST ran qPCR testing and associated data analysis.

$A E, K V$, and JS were pivotal in study design and conception. All authors have approved the manuscript.

\section{Funding}

Funding for this research was provided by Merck \& CO., Inc., Kenilworth, NJ,

USA.

Acknowledgments: The authors would like to thank Gokul Swaminathan for his critical analysis of the paper and MRL West Point Lab Animal Resources for their assistance with the animal studies.

\section{References}

1.Beagley KW, Timms P. Chlamydia trachomatis Infection: Incidence, Health Costs and Prospects for Vaccine Development. Journal of Reproductive Immunology. 2000;48:47-68.

2.Brunham RC, Rey-Ladino J. Immunology of Chlamydia infection: implications for a Chlamydia trachomatis vaccine. Nat Rev Immunol. 2005;5(2):149-61.

3.Morrison RP, Caldwell HD. Immunity to Murine Chlamydial Genital Infection. Infect Immun. 2002;70(6):2741-51. 
4.Gerbase AC, Rowley JT, Heymann DHL, Berkley SFB, Piot P. Global Pervalence and Incidence Estimates of Selected Curable STDs. Sexually Transmitted Infections. 1998;74(Suppl 1):S12-S6.

5.CDC. Chlamydia Screening Among Sexually Active Young Female Enrollees of Health Plans-- United States, 1999-2001. Morbidity and Mortality Weekly Report. 2004;53(42):983-5.

6.Pal S, Favaroni A, Tifrea DF, Hanisch PT, Luczak SE, Hegemann JH, et al. Comparison of the nine polymorphic membrane proteins of Chlamydia trachomatis for their ability to induce protective immune responses in mice against a C. muridarum challenge. Vaccine. 2017;35(19):2543-9.

7.Pal S, Peterson EM, Rappuoli R, Ratti G, de la Maza LM. Immunization with the Chlamydia trachomatis major outer membrane protein, using adjuvants developed for human vaccines, can induce partial protection in a mouse model against a genital challenge. Vaccine. 2006;24(6):766-75.

8.Pal S, Tatarenkova OV, de la Maza LM. A vaccine formulated with the major outer membrane protein can protect $\mathrm{C} 3 \mathrm{H} / \mathrm{HeN}$, a highly susceptible strain of mice, from a Chlamydia muridarum genital challenge. Immunology. 2015;146(3):432-43.

9.Yu H, Jiang X, Shen C, Karunakaran KP, Brunham RC. Novel Chlamydia muridarum T cell antigens induce protective immunity against lung and genital tract infection in murine models. J Immunol. 2009;182(3):1602-8.

10.Yu H, Jiang X, Shen C, Karunakaran KP, Jiang J, Rosin NL, et al. Chlamydia muridarum T-cell antigens formulated with the adjuvant DDA/TDB induce immunity against infection that correlates with a high frequency of gamma interferon (IFN-gamma)/tumor necrosis factor alpha and IFN-gamma/interleukin17 double-positive CD4+ T cells. Infection and immunity. 2010;78(5):2272-82.

11.Yu H, Karunakaran KP, Jiang X, Brunham RC. Evaluation of a multisubunit recombinant polymorphic membrane protein and major outer membrane protein $\mathrm{T}$ cell vaccine against Chlamydia muridarum genital infection in three strains of mice. Vaccine. 2014;32(36):4672-80.

12.Yu H, Karunakaran KP, Jiang X, Brunham RC. Subunit vaccines for the prevention of mucosal infection with Chlamydia trachomatis. Expert review of vaccines. 2016;15(8):977-88.

13.Karunakaran KP, Yu H, Jiang X, Chan Q, Moon KM, Foster LJ, et al. Outer membrane proteins preferentially load MHC class II peptides: implications for a Chlamydia trachomatis T cell vaccine. Vaccine. 2015;33(18):2159-66.

14.Ralli-Jain P, Tifrea D, Cheng C, Pal S, de la Maza LM. Enhancement of the protective efficacy of a Chlamydia trachomatis recombinant vaccine by combining systemic and mucosal routes for immunization. Vaccine. 2010;28(48):7659-66.

15.Vicetti Miguel RD, Quispe Calla NE, Pavelko SD, Cherpes TL. Intravaginal Chlamydia trachomatis Challenge Infection Elicits TH1 and TH17 Immune Responses in Mice That Promote Pathogen Clearance 
and Genital Tract Damage. PLOS ONE. 2016;11(9):e0162445.

16.Scurlock AM, Frazer LC, Andrews CW, Jr., O’Connell CM, Foote IP, Bailey SL, et al. Interleukin-17 contributes to generation of Th1 immunity and neutrophil recruitment during Chlamydia muridarum genital tract infection but is not required for macrophage influx or normal resolution of infection. Infection and immunity. 2011;79(3):1349-62.

17.0'Meara CP AC, Harvie MC, Andrew DW, Timms P, Lycke NY, Beagley KW. Immunity against a Chlamydia infection and disease may be determined by a balance of IL-17 signaling. Immunology and Cell Biology. 2014;92:287-97.

18.Pal S, Theodor I, Peterson EM, de la Maza LM. Immunization with an acellular vaccine consisting of the outer membrane complex of Chlamydia trachomatis induces protection against a genital challenge. Infection and immunity. 1997;65(8):3361-9.

19.Lindenstrom T, Woodworth J, Dietrich J, Aagaard C, Andersen P, Agger EM. Vaccine-induced th17 cells are maintained long-term postvaccination as a distinct and phenotypically stable memory subset. Infection and immunity. 2012;80(10):3533-44.

20.Swanson KA, Taylor LD, Frank SD, Sturdevant GL, Fischer ER, Carlson JH, et al. Chlamydia trachomatis polymorphic membrane protein $\mathrm{D}$ is an oligomeric autotransporter with a higher-order structure. Infection and immunity. 2009;77(1):508-16.

21. Wooters MA, Kaufhold RM, Field JA, Indrawati L, Heinrichs JH, Smith JG. A real-time quantitative polymerase chain reaction assay for the detection of Chlamydia in the mouse genital tract model. Diagnostic microbiology and infectious disease. 2009;63(2):140-7.

22.Morrison R, K. Feilzer, Tumas DB. Gene knockout mice establish a primary protective role for major histocompatibility complex class II-restricted responses in Chlamydia trachomatis genital tract infection. Infect Immun. 1995;63(12):4661-8.

23.Morrison SG, Morrison RP. Resolution of secondary Chlamydia trachomatis genital tract infection in immune mice with depletion of both CD4+ and CD8+ T cells. Infection and immunity. 2001;69(4):2643-9.

24.Morrison SG, Su H, Caldwell HD, Morrison RP. Immunity to murine Chlamydia trachomatis genital tract reinfection involves $B$ cells and CD4(+) T cells but not CD8(+) T cells. Infection and immunity. 2000;68(12):6979-87.

25.Rank RG, Soderberg LS, Barron AL. Chronic chlamydial genital infection in congenitally athymic nude mice. Infection and immunity. 1985;48(3):847-9.

26.Lu C, Zeng H, Li Z, Lei L, Yeh IT, Wu Y, et al. Protective immunity against mouse upper genital tract pathology correlates with high IFNgamma but low IL-17 T cell and anti-secretion protein antibody responses induced by replicating chlamydial organisms in the airway. Vaccine. 2012;30(2):475-85. 
27.Yu H, Karunakaran KP, Kelly I, Shen C, Jiang X, Foster LJ, et al. Immunization with live and dead Chlamydia muridarum induces different levels of protective immunity in a murine genital tract model: correlation with MHC class II peptide presentation and multifunctional Th1 cells. J Immunol. 2011;186(6):3615-21.

28.Farris CM, Morrison SG, Morrison RP. CD4+ T cells and antibody are required for optimal major outer membrane protein vaccine-induced immunity to Chlamydia muridarum genital infection. Infection and immunity. 2010;78(10):4374-83.

29.Igietseme JU, Rank RG. Susceptibility to reinfection after a primary chlamydial genital infection is associated with a decrease of antigen-specific T cells in the genital tract. Infection and immunity. 1991;59(4):1346-51.

30.Morrison SG, Morrison RP. A predominant role for antibody in acquired immunity to chlamydial genital tract reinfection. J Immunol. 2005;175(11):7536-42.

31.Perry LL, Feilzer K, Caldwell HD. Immunity to Chlamydia trachomatis is mediated by T helper 1 cells through IFN-gamma-dependent and -independent pathways. J Immunol. 1997;158(7):3344-52.

32.Roshick C, Wood H, Caldwell HD, McClarty G. Comparison of gamma interferon-mediated antichlamydial defense mechanisms in human and mouse cells. Infection and immunity. 2006;74(1):225-38.

33.Swaminathan G, Thoryk EA, Cox KS, Meschino S, Dubey SA, Vora KA, et al. A novel lipid nanoparticle adjuvant significantly enhances $B$ cell and $T$ cell responses to sub-unit vaccine antigens. Vaccine. 2016;34(1):110-9.

34.Klinguer-Hamour C, Libon C, Plotnicky-Gilquin H, Bussat MC, Revy L, Nguyen T, et al. DDA adjuvant induces a mixed Th1/Th2 immune response when associated with BBG2Na, a respiratory syncytial virus potential vaccine. Vaccine. 2002;20(21-22):2743-51.

\section{Figures}

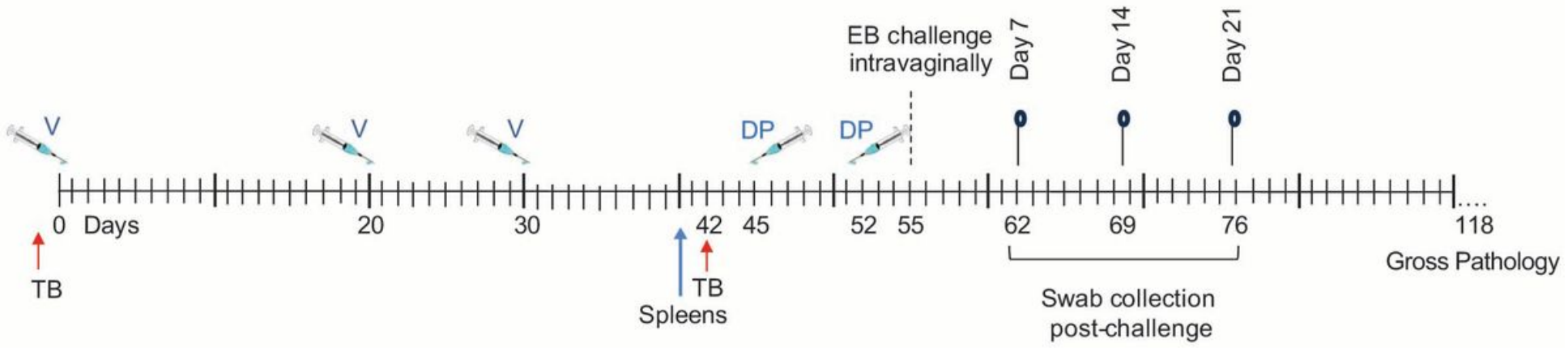

Figure 1 
Study Design Schedule of vaccination, collection of spleens, Chlamydia muridarum challenge, cervicovaginal swabbing, and gross pathology in female C57BL/6 mice. V, vaccination; DP, depo-provera treatment; TB, tail bleeds; Spleens, harvested for ICS.

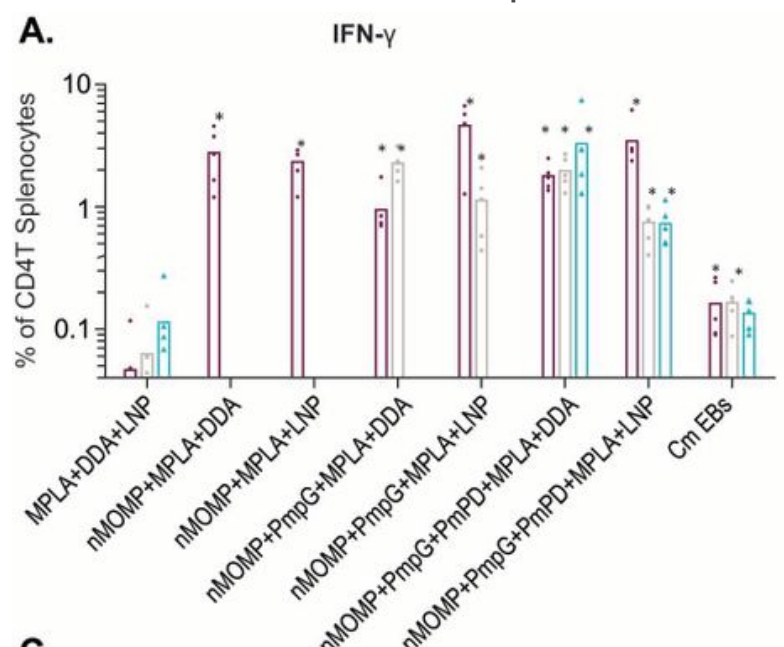

B. $\quad$ TNFa

C.
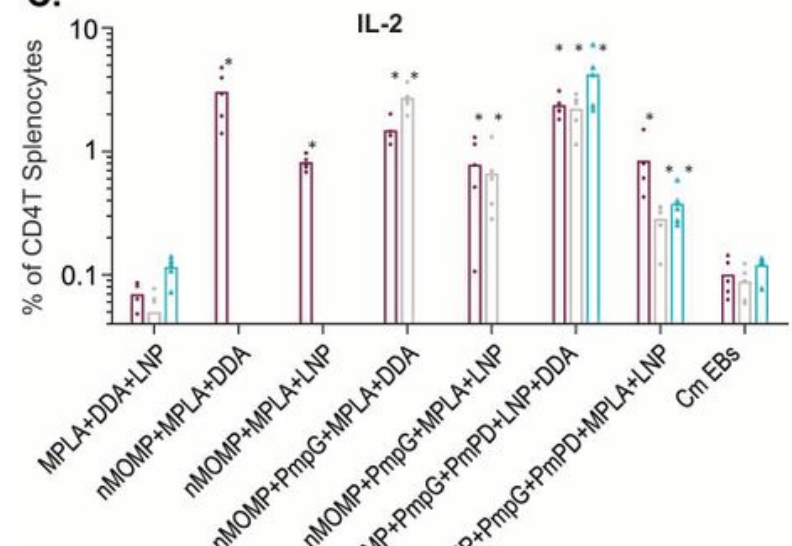

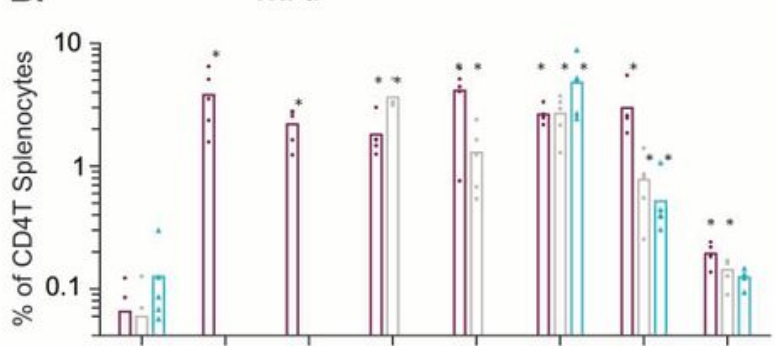

D.
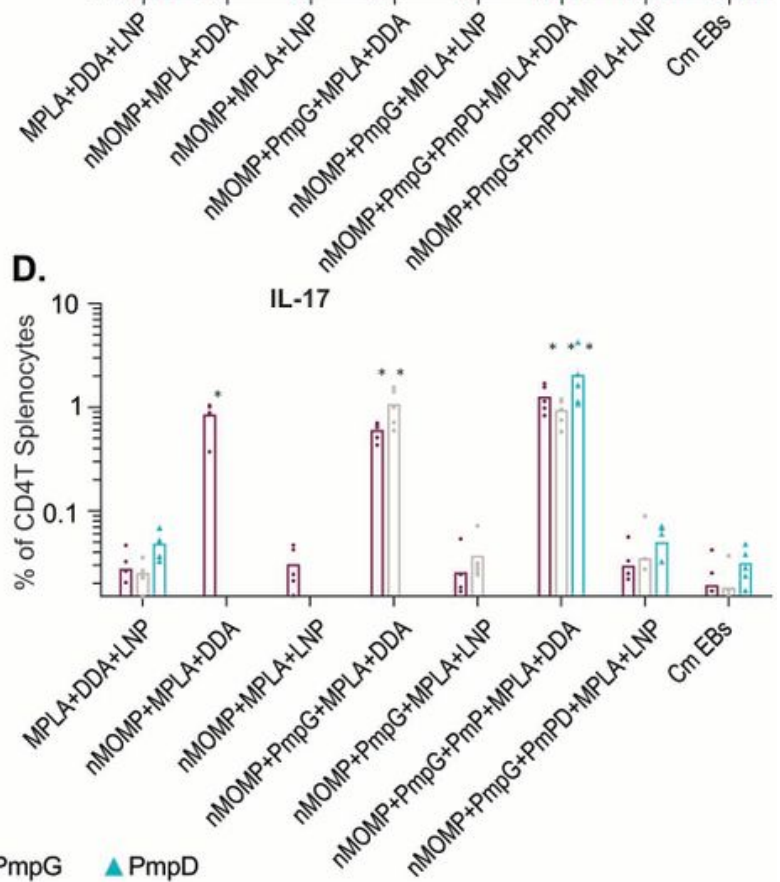

E.

- MOMP $=\mathrm{PmpG} \triangle \mathrm{PmpD}$

DDA+ MPLA +

TNP+MPLA+

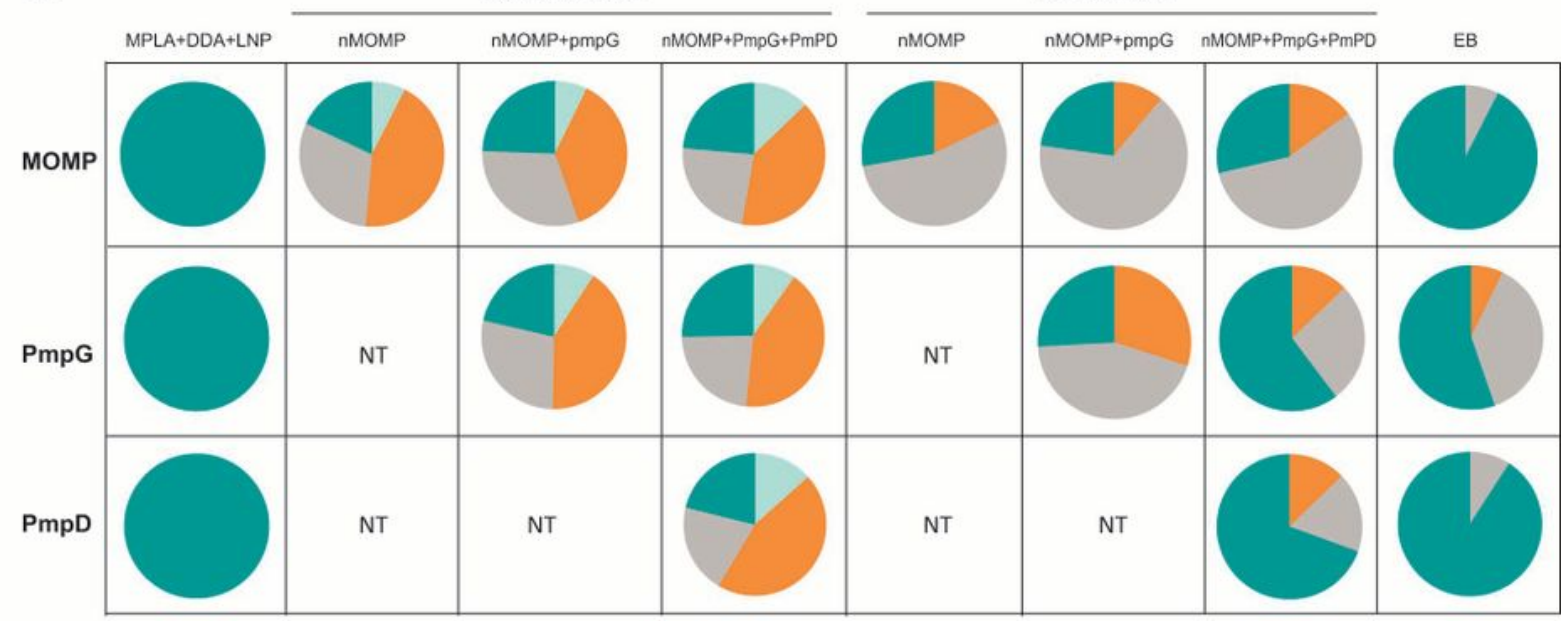

$\mathrm{IFNy}+\mathrm{IL} 2+\mathrm{TNFa}+\mathrm{IL} 17+$ cells

$3+$ cytokines

$2+$ cytokines

$1+$ cytokines

Figure 2

FACS Antigen specific cytokine responses after immunization with Chlamydia proteins formulated with adjuvants. Splenocytes from the adjuvant control group, vaccine immunized groups and Cm EB 
vaccinated group were stimulated with 15 mer peptide pools corresponding to the vaccine antigens for each group (MOMP, PmpG, PmpD). Bar charts represent the percent of CD4 T cells producing IFN- $\gamma(2 A)$, TNF-a (2B), IL-2 (2C) or IL-17 (2D) as evaluated by multiparameter flow cytometry. Individual points within each open bar graph represent data from an individual mouse, and the height of the open bar depicts the mean response for the group. * depicts a significant difference $(p<0.05)$ in response as compared to the adjuvant only group (MPLA+DDA+LNP) in a two tailed paired $t$ test. NT, not tested.

A.
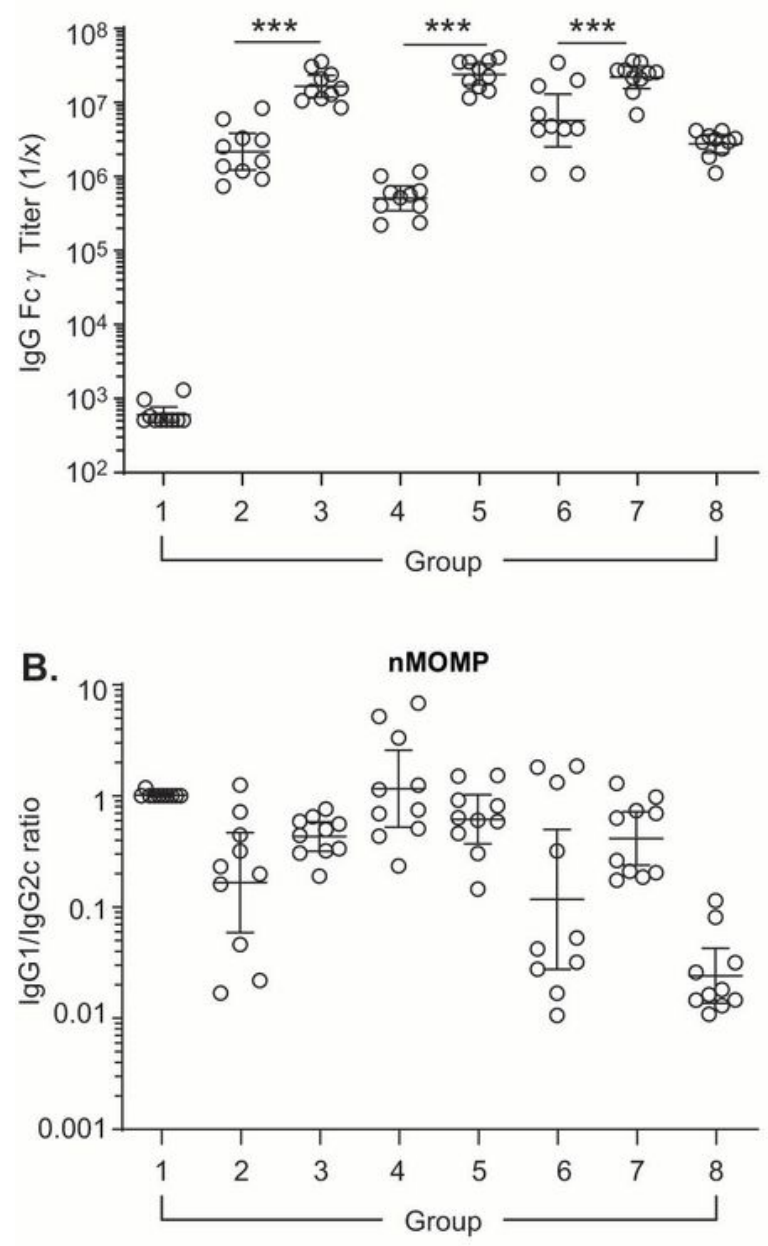

c.

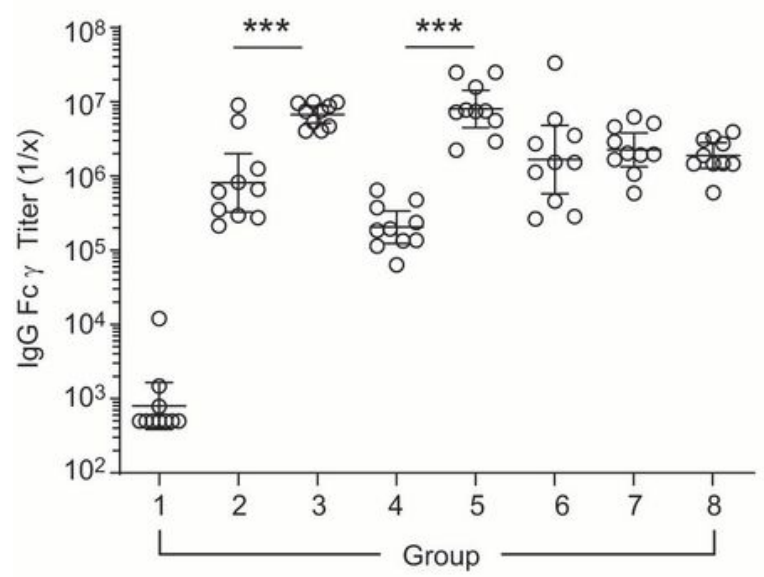

D.
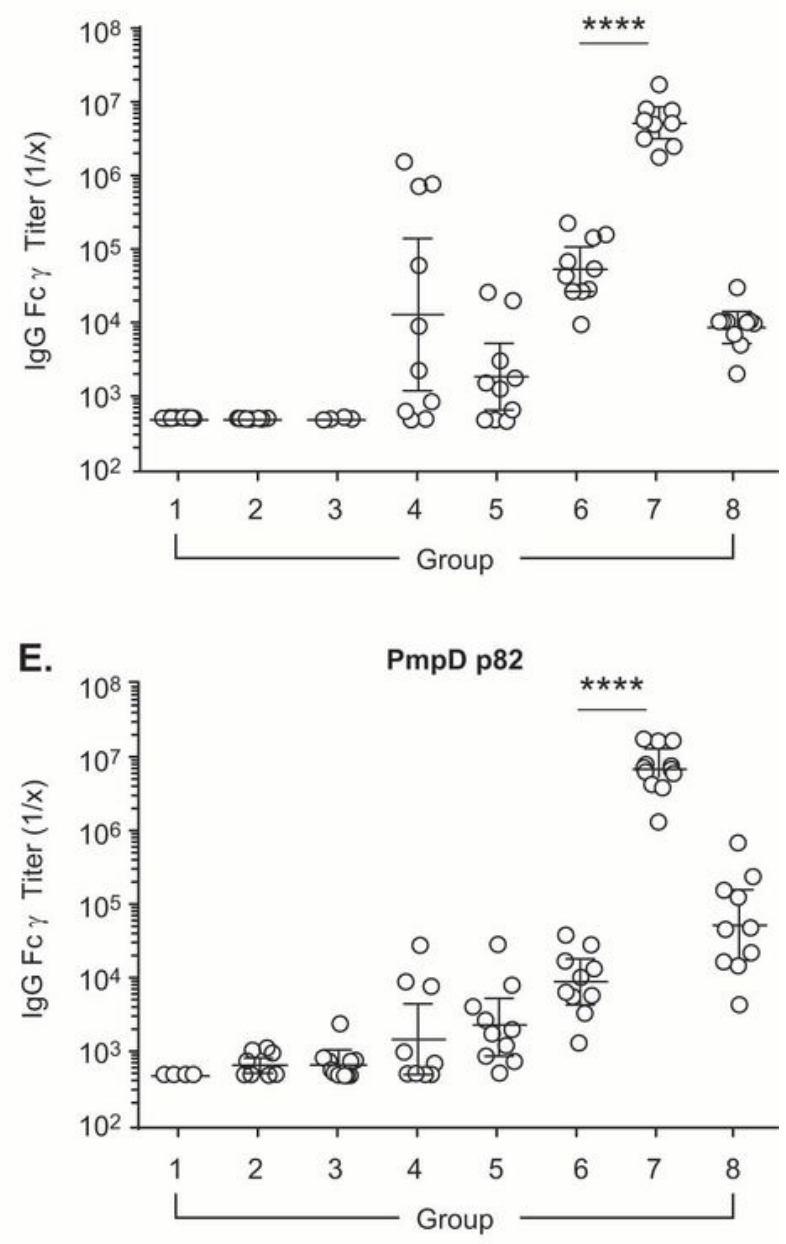

F.

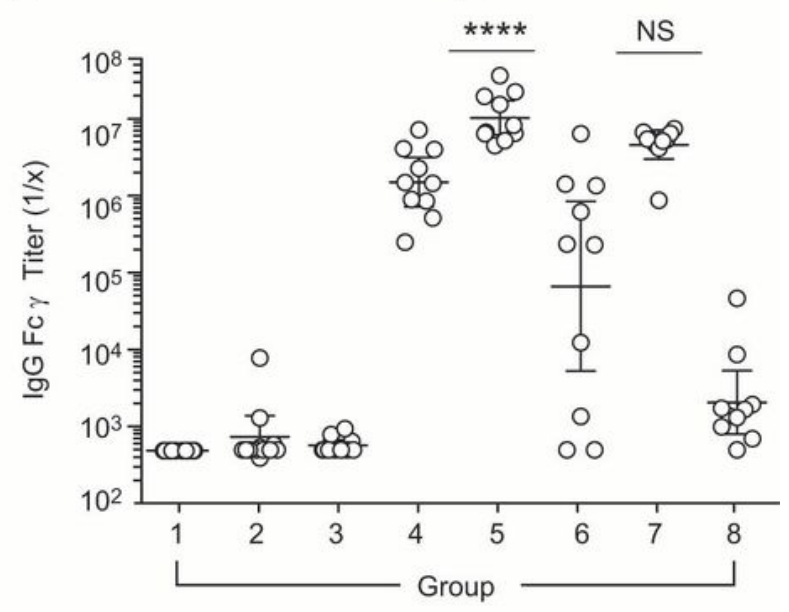

Figure 3 
ELISA IgG antibody titers to $\mathrm{nMOMP}, \mathrm{EB}, \mathrm{PmpG}$, and PmpD as determined by ELISA. 3A. Total IgG titers to nMOMP. 3B. IgG1/lgG2c ratio of anti-nMOMP titers. 3C. Total IgG titers to $C$. muridarum EBs 3D. Total IgG titers to PmpD p73 3E. Total IgG titers to PmpD p82, 3F. Total IgG titers to PmpG. Grp 1) MPLA+DDA+LNP, Grp 2) $\mathrm{Cm}$ nMOMP+MPLA+DDA, Grp 3) Cm nMOMP+MPLA+LNP, Grp 4) $\mathrm{Cm}$ nMOMP+PmpG+MPLA+DDA, Grp 5) Cm nMOMP+PmpG+MPLA+LNP, Grp 6) Cm nMOMP+PmpG+PmpD p73+PmpD p82+MPLA+DDA, Grp 7) Cm nMOMP+PmpG+PmpD p73+PmpD p82+MPLA+LNP, Grp 8) C. muridarum EBs. Error bars are geometric means with $95 \%$ confidence intervals. Transformed data analyzed by One-way ANOVA with Dunnett post test * $p<0.05$, ${ }^{\star *} p<0.01,{ }^{* \star *} p<0.001$, ${ }^{* \star \star *} p<0.0001$, NS not significant 


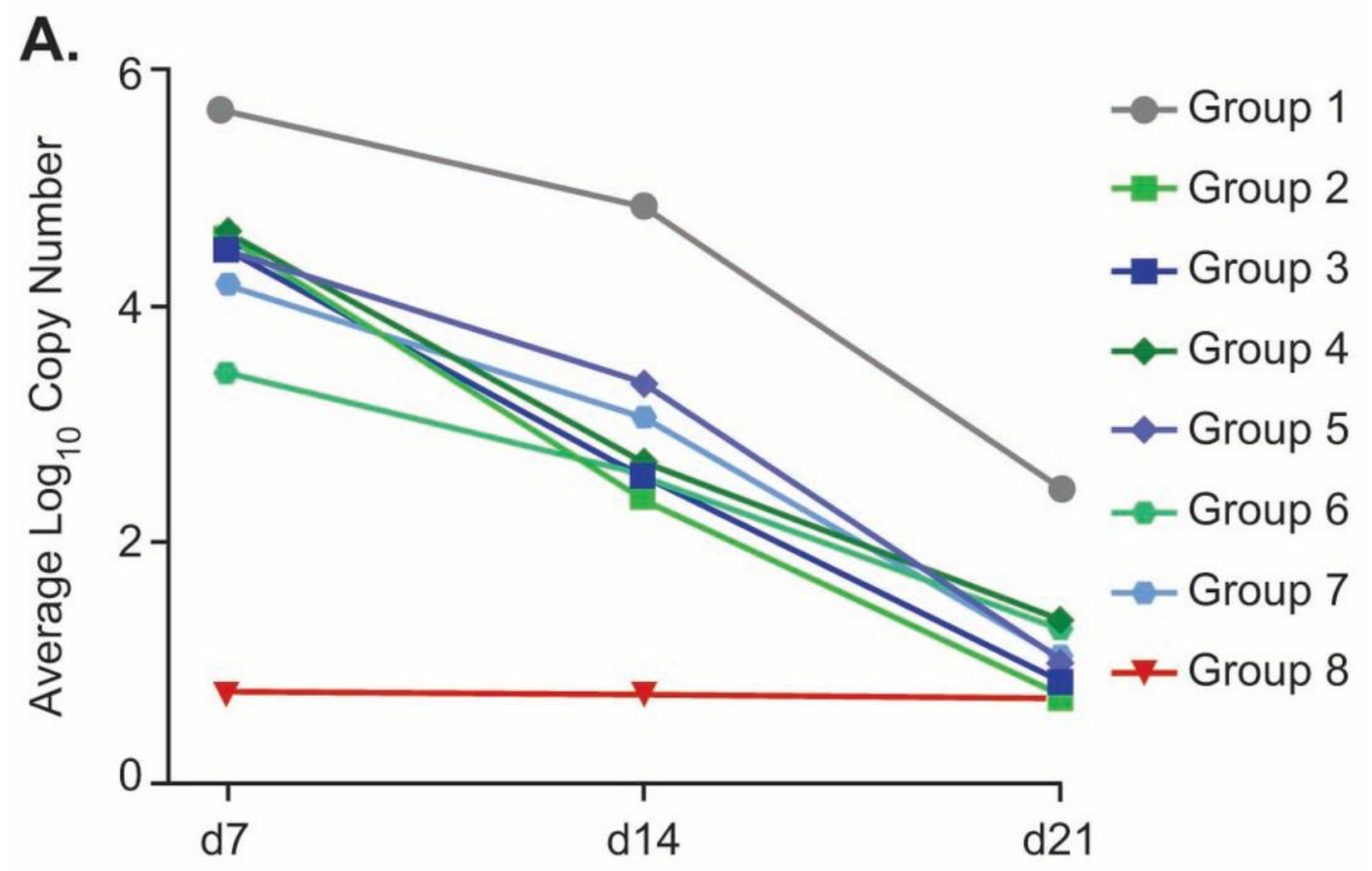

B.

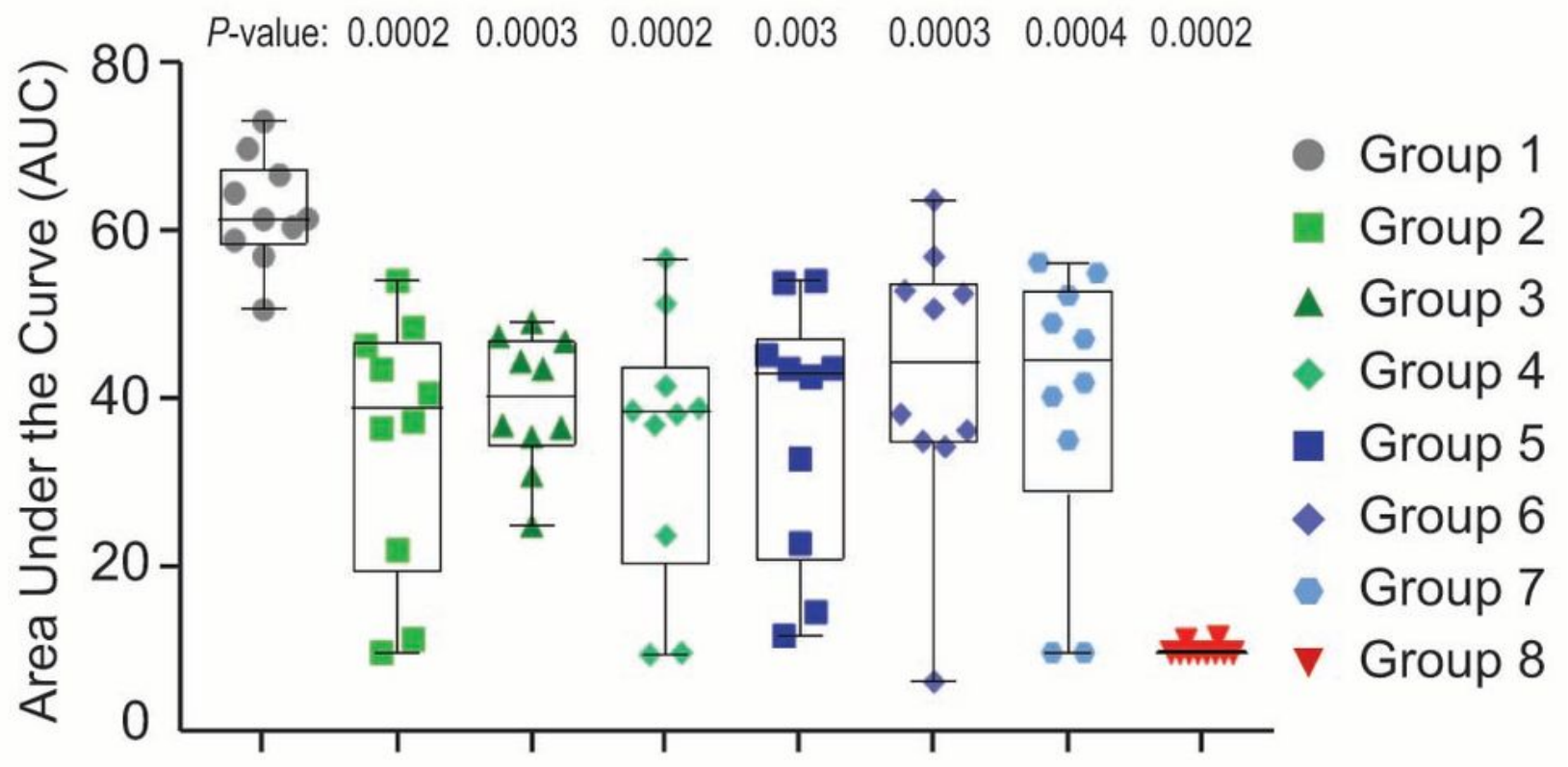

Figure 4

Chlamydia muridarum challenge in mice. 4A.) Average copy numbers from real-time qPCR for each vaccine test group were plotted on Log10 scale over days post challenge. 4B.) Area under the curve (AUC) values for individual mice were calculated and plotted and further analyzed by Wilcoxon Rank Sum Test to determine the $p$-values for each group (compared to MPLA/DDA/LNP group). Grp 1) MPLA+DDA+LNP, Grp 2) Cm nMOMP+MPLA+DDA, Grp 3) Cm nMOMP+MPLA+LNP, Grp 4) Cm 
nMOMP+PmpG+MPLA+DDA, Grp 5) Cm nMOMP+PmpG+MPLA+LNP, Grp 6) Cm nMOMP+PmpG+PmpD p73+PmpD p82+MPLA+DDA, Grp 7) Cm nMOMP+PmpG+PmpD p73+PmpD p82+MPLA+LNP, Grp 8) C. muridarum EBs.

\section{Supplementary Files}

This is a list of supplementary files associated with this preprint. Click to download.

- NC3RsARRIVEGuidelinesChecklistfillablefinal220ct.pdf 